\title{
CONCERNING CONTINUITY APART FROM A MEAGER SET
}

\author{
JANUSZ KANIEWSKI AND ZBIGNIEW PIOTROWSKI
}

ABSTRACT. Given a $\sigma$-ideal $\mathscr{I}$ of subsets of a space $X$, mappings $f: X \rightarrow Y$ are investigated, such that $f \mid X_{0}$ is continuous for some closed $X_{0} \subset X$ with $X \backslash X_{0} \in \mathscr{I}$.

1. Introduction. Throughout the paper, $X$ and $Y$ are topological spaces, and $\mathscr{I}$ is a $\sigma$-ideal of subsets of $X$.

The following theorem was first proved by Kuratowski in 1930. (See [K1] and [KM, p. 408]; also [EFK] for a closely related result.)

If $Y$ is second countable, then the statement

(a) $f \mid X_{0}$ is continuous for some $X_{0} \subset X$ with $X \backslash X_{0} \in \mathscr{I}$ is equivalent to

(b) for every open $V \subset Y, f^{-1}(V)=(U \backslash A) \cup B$, where $U$ is open in $X, A \in \mathscr{I}$, and $B \in \mathscr{I}$.

We are interested in characterizing mappings that admit continuous restriction to a set $X_{0} \subset X$ with an extra property in addition to $X \backslash X_{0} \in \mathscr{I}$. The present paper is to investigate the case of a closed $X_{0}$.

2. The theorem. It is an easy observation that if then

(i) $f \mid X_{0}$ is continuous for some closed $X_{0} \subset X$ with $X \backslash X_{0} \in \mathscr{I}$,

(ii) for every open $V \subset Y, f^{-1}(V)=U \backslash A$ with $U$ open in $X$ and $A \in \mathscr{I}$.

Indeed, let $X_{0}$ be as in (i) and take any closed $F \subset Y$. For (ii) to hold true, $f^{-1}(F)$ should have a form of $E \cup A$ with $E \subset X$ closed and $A \in \mathscr{I}$. Since

$$
\begin{aligned}
f^{-1}(F) & =\left[f^{-1}(F) \cap X_{0}\right] \cup\left[f^{-1}(F) \cap\left(X \backslash X_{0}\right)\right] \\
& =\left(f \mid X_{0}\right)^{-1}(F) \cup\left[f^{-1}(F) \cap\left(X \backslash X_{0}\right)\right],
\end{aligned}
$$

this is indeed the case.

We are now going to describe circumstances where (i) and (ii) are actually equivalent. To make some statements shorter, let us agree to say that $f$ is $\mathscr{A}_{\text {continu- }}$ ous whenever (ii) is satisfied.

THEOREM. Let $Y$ be a regular space and assume that either

(I) $Y$ is second countable, or

(II) $X$ is hereditarily Lindelöf, or

(III) $\mathscr{I}$ consists of all meager sets in $X$.

Received by the editors June 24, 1985 and, in revised form, September 26, 1985.

1980 Mathematics Subject Classification (1985 Revision). Primary 54C30; Secondary 26 A15. 
Let $f: X \rightarrow Y$ be A-continuous. Then $f \mid X_{0}$ is continuous for some closed $X_{0} \subset X$ with $X \backslash X_{0} \in \mathscr{I}$.

3. Examples. Before a proof, let us look at two examples showing that the hypotheses of the theorem are to some extent indispensable; namely that

in (I), "second countable" cannot be weakened to "Lindelöf",

in (II), "hereditarily Lindelöf" cannot be weakened to "Lindelöf",

in (III), $\mathscr{I}$ cannot be just any $\sigma$-ideal, and regularity of $Y$ is essential in all three versions.

A. Let $X=Y=\mathbf{R}$ with topologies as follows. All points except for 0 are isolated in both spaces, while neighborhoods of 0 in $X$ (respectively, in $Y$ ) are complements of finite (respectively, countable) sets. These spaces $X$ and $Y$ are known to have nice topological properties: $X$ is compact Hausdorff, $Y$ is regular Hausdorff and Lindelöf.

Let $\mathscr{I}$ be the $\sigma$-ideal of all countable subsets of $X$. Although the identity function $f: X \rightarrow Y$ is obviously $\mathscr{A}$ continuous, its restriction to any closed, co-countable set $X_{0} \subset X$ is discontinuous at 0 .

B. Now consider $X=\mathbf{R}$ with its usual topology. Let $Y=\mathbf{R}$ have a richer topology consisting of all sets $U \backslash A$, where $U$ is in the usual topology of $\mathbf{R}$ and $A$ is a subset of $Z=\{1 / n: n \in \mathbf{N}\}$. Thus $Y$ is Hausdorff and second countable.

Let $\mathscr{I}$ be the $\sigma$-ideal of, say, all meager sets in $X$ (or all sets of Lebesgue measure 0 , or all countable sets). Since $Z \in \mathscr{I}$, the identity function $f: X \rightarrow Y$ is $\mathscr{A}$-continuous. On the other hand, the only closed set in $X$ whose complement is a member of $\mathscr{I}$ is $X$ itself. Therefore, the only restriction of $f$ to be considered is $f$ itself, and that is not continuous.

4. Proof of the theorem. In case (I), let $\left\{V_{n}: n \in \mathbf{N}\right\}$ be a base for the topology of

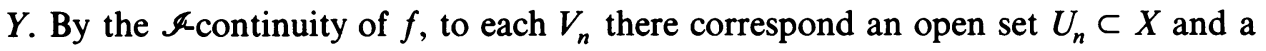
set $A_{n} \in \mathscr{I}$ so that

\section{Put}

$$
f^{-1}\left(V_{n}\right)=U_{n} \backslash A_{n}
$$

and

$$
I=\bigcup\left\{A_{n}: n \in \mathbf{N}\right\}
$$

Of course, $X_{0}$ is closed in $X$ and $X \backslash X_{0} \subset I \in \mathscr{I}$. To show that $f \mid X_{0}$ is continuous, take any $x \in X_{0}$ and any neighborhood $V$ of $f(x)$ in $Y$. Since $Y$ is regular, there is an $n \in \mathbf{N}$ such that $f(x) \in V_{n}$ and

$$
\mathrm{cl} V_{n} \subset V \text {. }
$$

We will show that the corresponding neighborhood $U_{n}$ of $x$ in $X$, defined by (1), satisfies $f\left(U_{n} \cap X_{0}\right) \subset V$; this will prove that $f \mid X_{0}$ is continuous at $x$.

Suppose that, on the contrary, $f\left(x^{\prime}\right) \notin V$ for some $x^{\prime} \in U_{n} \cap X_{0}$. Then, by (4), $f\left(x^{\prime}\right) \in Y \backslash \operatorname{cl} V_{n}$ and so, by the definition of a base, there is a $V_{m}$ with $f\left(x^{\prime}\right) \in V_{m}$ and

$$
V_{m} \cap V_{n}=\varnothing
$$


The corresponding set $U_{m}$ contains $x^{\prime}$; therefore

$$
x^{\prime} \in U_{m} \cap U_{n} \cap X_{0}
$$

On the other hand, (1) and (2) yield $U_{n} \subset f^{-1}\left(V_{n}\right) \cup I$ and likewise, $U_{m} \subset f^{-1}\left(V_{m}\right) \cup$ I. Hence, in view of (4),

$$
U_{n} \cap U_{m} \subset f^{-1}\left(V_{n} \cap V_{m}\right) \cup I=I
$$

and consequently, $U_{n} \cap U_{m} \subset$ int $I$. Now (3) gives $U_{n} \cap U_{m} \cap X_{0}=\varnothing$, a contradiction to (6).

Cases (II) and (III) can be given a common proof. But first, let us recall a theorem known as Banach Category Theorem. (See [B] and [O, p. 62].)

If a subset $Z \subset X$ is locally meager then $Z$ is meager.

An immediate corollary to the Banach Category Theorem is the following:

The union of all subsets of $X$ that are open and meager at the same time is meager.

In other words, in case (III) of the theorem, the following condition (*) is satisfied:

(*) The union of all members of $I$ that are open in $X$ is a member of $I$.

The above holds in case (II) as well, since the union of all such sets (in a hereditarily Lindelöf space) coincides with the union of just countably many of them, which is in $\mathscr{I}$ by the definition of a $\sigma$-ideal. Thus (*) is a property that (II) and (III) have in common.

Let us say that $f$ is pointwise acontinuous if, for every $x \in X$ and for every neighborhood $V$ of $f(x)$ in $Y$, there exists a neighborhood $U$ of $x$ in $X$ such that $U \backslash f^{-1}(V) \in \mathscr{I}$.

Obviously, every $\mathbb{A}$-continuous mapping is pointwise $\mathbb{A}$ continuous. Therefore, to complete a proof of the theorem, it will suffice to prove the following lemma.

Lemma. Let $Y$ be a regular space and let $f$ be pointwise $\mathbb{A}$-continuous. Put

$$
X_{0}=X \backslash \cup\{G: G \text { is open in } X \text { and } G \in \mathscr{I}\} \text {. }
$$

Then $f \mid X_{0}$ is continuous.

Proof. Consider any point $x \in X_{0}$ and any neighborhood $V$ of $f(x)$ in $Y$. We need a neighborhood $U$ of $x$ in $X$ such that $f\left(U \cap X_{0}\right) \subset V$.

First, using regularity of $Y$, we can select an open set $W$ containing $f(x)$ with

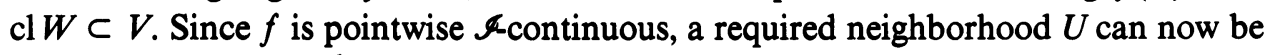
chosen so that $U \backslash f^{-1}(W) \in \mathscr{G}$.

To show that $f\left(U \cap X_{0}\right) \subset V$, take any $x^{\prime} \in U \cap X_{0}$; since cl $W \subset V$, it will be enough to prove that $f\left(x^{\prime}\right) \in \mathrm{cl} W$.

Suppose that, on the contrary, there is a neighborhood $W^{\prime}$ of $f\left(x^{\prime}\right)$ such that $W \cap W^{\prime}=\varnothing$. By the pointwise $\mathscr{G}$ continuity of $f$, we have $U^{\prime} \backslash f^{-1}\left(W^{\prime}\right) \in \mathscr{I}$ for some neighborhood $U^{\prime}$ of $x^{\prime}$. Hence

$$
U \cap U^{\prime}=\left(U \cap U^{\prime}\right) \backslash f^{-1}\left(W \cap W^{\prime}\right) \subset\left[U \backslash f^{-1}(W)\right] \cup\left[U^{\prime} \backslash f^{-1}\left(W^{\prime}\right)\right] \in \mathscr{I},
$$

and therefore $U \cap U^{\prime} \subset X \backslash X_{0}$, by the definition of $X_{0}$.

We have reached a contradiction, because on the other hand, $x^{\prime} \in U \cap U^{\prime} \cap X_{0}$. 
5. $\mathscr{A}$-continuity versus pointwise $\mathscr{A}$-continuity. As noted, every $\mathscr{A}$-continuous mapping is pointwise $\mathscr{A}$-continuous. The converse is not true in general.

For example, let $X=X_{1} \times X_{2}$, where $X_{1}$ is a discrete uncountable space and $X_{2}=\{0,1,1 / 2,1 / 3, \ldots\}$ with the usual topology. Let $\mathscr{I}$ be the $\sigma$-ideal of all countable subsets of $X$. Since the space $X$ is locally countable, any mapping defined on it will automatically be pointwise $\mathscr{A}$-continuous. Not necessarily $\mathscr{A}$-continuous, though, as witnessed by the characteristic function of the set $X_{1} \times\{0\}$. Notice that $(*)$ is not satisfied in this example.

For another example, let $X$ and $\mathscr{I}$ be same as above, ${ }^{1}$ except that $X_{2}$ is now $\mathbf{R}$ with the usual topology. Let $Y$ be as in the second example of $\S 3$. It is easy to see that the mapping $f\left(x_{1}, x_{2}\right)=x_{2}$ is pointwise $\mathscr{I}$-continuous but not $\mathscr{I}$-continuous, although (*) is now satisfied in $X$.

However, given both (*) in $X$ and regularity of $Y$, every pointwise $\mathscr{f}$-continuous mapping $f: X \rightarrow Y$ is $\mathscr{A}$ continuous. This follows immediately from the lemma of $\S 4$ and from the observation of $\$ 2$.

The same is true regardless of regularity of $Y$, provided (*) is replaced with the following stronger condition.

(**) For every set $A \subset X$, if each point $x \in A$ has a neighborhood $U(x)$ such that $U(x) \cap A \in \mathscr{I}$, then $A \in \mathscr{I}^{2}$

More precisely, (**) implies that

(T) the family $\mathscr{T}=\{U \backslash A: U$ is open in $X, A \in \mathscr{I}\}$ is closed under arbitrary unions, ${ }^{3}$

which, in turn, implies that every pointwise $\mathscr{A}$ continuous mapping defined on $X$ is A-continuous.

Indeed, assume (**) and consider any union $E=\bigcup\left\{U_{s} \backslash A_{s}: s \in S\right\}$ of members of $\mathscr{T}$. Put $U=\bigcup\left\{U_{s}: s \in S\right\}$ and $A=U \backslash E$. Since each $x \in A$ is in some $U_{s}$ and since $U_{s} \cap A \subset A_{s} \in \mathscr{I}$, the condition (**) yields $A \in \mathscr{I}$, and therefore $E=U \backslash A$ $\in \mathscr{T}$. The other implication is equally straightforward.

6. An application. In 1923, Sierpiński and Zygmund [SZ] defined a function $h$ : $\mathbf{R} \rightarrow \mathbf{R}$ whose restriction to every set of power $c$ is not continuous. The following is a more general setting of their result. (Compare [K2, p. 422].)

For every metric separable space $X$, and for every complete metric space $Y$ of power $c$, there exists a mapping $h: X \rightarrow Y$ whose restriction to every set of power $c$ is not continuous.

The theorem of this paper enables us to further improve that result as follows.

Corollary. Let $X$ be a metric separable space and let $Y$ be a complete metric space. Assume that $\mathscr{I}$ contains all subsets of $X$ having power $<c$. Then there exists $a$ mapping $h: X \rightarrow Y$ such that for every set $X_{0} \subset X$ with $X_{0} \notin \mathscr{I}$, the restriction $f \mid X_{0}$ is not $\mathscr{I}_{0}$-continuous, $\mathscr{I}_{0}$ being the o-ideal $\left\{A: A \in \mathscr{I}, A \subset X_{0}\right\}$.

\footnotetext{
${ }^{1}$ Alternatively, $\mathscr{I}$ can be the $\sigma$-ideal of all meager sets in $X$.

${ }^{2}$ This condition is still met when either $\mathscr{I}=$ meager sets (Banach Category Theorem), or $X$ is a hereditarily Lindelöf space.

${ }^{3}$ And therefore $\mathscr{T}$ satisfies the axioms for a topology in $X$; Acontinuity then means continuity with respect to that richer topology in $X$.
} 
Proof. Let $h$ be the mapping defined by Sierpiński and Zygmund. Suppose $h \mid X_{0}$ is $\mathscr{I}_{0}$-continuous for some $X_{0} \subset X$ with $X_{0} \notin \mathscr{I}$. According to our theorem (case (II)), there is a set $X_{1} \subset X_{0}$ such that $X_{0} \backslash X_{1} \in \mathscr{I}_{0}$ and $h \mid X_{1}$ is continuous. By the choice of $h$, this set $X_{1}$ has power $<c$. Therefore $X_{1} \in \mathscr{I}$, and consequently $X_{0}=\left(X_{0} \backslash X_{1}\right) \cup X_{1} \in \mathscr{I}$. This contradiction ends the proof.

A particular case can be obtained by letting $\mathscr{I}$ contain all sets of power $<c$ and no other sets. ${ }^{4}$ Then the property of the function $h$ reads as follows.

Every set $X_{0}$ of power c contains a point $x_{0}$ such that $h$ takes c-many points of every neighborhood of $x_{0}$ in $X_{0}$ outside some fixed neighborhood of $f\left(x_{0}\right)$.

7. Remarks. A. In the case when $\varnothing$ is the only member of $\mathscr{I}$ that is open in $X$, for instance if $X$ is a Baire space and $\mathscr{I}$ is the $\sigma$-ideal of all meager subsets of $X$, condition (i) of $\$ 2$ coincides with continuity of $f$.

Also in that case, our lemma of $\$ 4$ states that every (pointwise) $\mathscr{A}$-continuous mapping $f: X \rightarrow Y$ is continuous, provided $Y$ is regular.

B. One might be tempted to expect the following two statements to be equivalent as well, at least under some reasonable assumptions on $X, Y$, and/or $\mathscr{I}$.

(i) $f \mid X_{0}$ is continuous for some open $X_{0} \subset X$ with $X \backslash X_{0} \in \mathscr{I}$,

(ii)' for every open $V \subset Y, f^{-1}(V)=U \cup A$ with $U$ open in $X$ and $A \in \mathscr{I}$.

As in \$2, (i)' implies (ii)' with no assumptions whatsoever. Unfortunately, the converse is false even when $X=Y=\mathbf{R}$ (with natural topology) and $\mathscr{I}$ is any of the standard $\sigma$-ideals in $\mathbf{R}$. To see this, arrange all rational numbers in a sequence $q_{1}$, $q_{2}, \ldots$ and put

$$
f(x)= \begin{cases}1 / n & \text { if } x=q_{n}, \\ 0 & \text { if } x \text { is irrational. }\end{cases}
$$

Let $\mathscr{I}$ be any proper $\sigma$-ideal containing all rationals. The inverse image of every open set $V \subset Y$ has a required form, since it is either open or a subset of the rationals. However, $f$ is discontinuous on every open nonempty set.

\section{REFERENCES}

[B] S. Banach, Théorème sur les ensembles de première catégorie, Fund. Math. 16 (1930), 395-398.

[EFK] A. Emeryk, R. Frankiewicz and W. Kulpa, On functions having the Baire property, Bull. Acad. Polon. Sci. Sér. Sci. Math. 27 (1979), 489-491.

[K1] K. Kuratowski, La propriété de Baire dans les espaces métriques, Fund. Math. 16 (1930), 390-394.

[K2] _ Topology, Vol. I, Academic Press; PWN, 1966.

[KM] K. Kuratowski and A. Mostowski, Set theory, 2nd ed., North-Holland, Amsterdam; PWN, Warsaw, 1976.

[O] J. C. Oxtoby, Measure and category, Springer-Verlag, 1971.

[SZ] W. Sierpiński and A. Zygmund, Sur une fonction qui est discontinue sur tout ensemble de puissance du continu, Fund. Math. 4 (1923), 316-318.

Department of Mathematics, Warsaw University, PKiN, 00 - 901 WarSaw, Poland

Department of Mathematics, Youngstown State University, Youngstown, Ohio 44555

\footnotetext{
${ }^{4}$ This $\mathscr{I}$ is a $\sigma$-ideal by König's theorem.
} 